\title{
Syphilis: case series
}

\section{Aryambika Krishnan, Aneesh Samayam, Anjan Kumar Patra}

\author{
Department of Dermatology, M.V.J Medical College and Research Hospital, Bangalore, India
}

Corresponding author: Dr. Aryambika Krishnan, E-mail: aryakr27@gmail.com

\begin{abstract}
Syphilis is a sexually transmitted infectious disease caused by Treponema pallidum. This case series reports 3 cases of syphilis and highlights the varied presentation of primary and secondary syphilis which is rare in present day clinical scenario and also the association of syphilis and HIV co-infection. Case 1: A case of primary syphilis presented with solitary painless genital ulcer, associated with lymphadenopathy and VDRL was reactive in 1:32 dilution. Case 2: A retro positive patient presented with primary and secondary syphilitic lesions manifesting as multiple genital ulcers, disseminated skin rashes and oral lesions. VDRL and HIV was reactive. Case 3: A case of secondary syphilis presented with hyperpigmented annular plaques over both palms and soles with healing genital ulcers. VDRL and HIV was reactive.
\end{abstract}

Key words: Syphilis; HIV; Treponema pallidum

\section{INTRODUCTION}

Syphilis is an infectious disease caused by Treponema pallidum. Transmission occurs through sexual contact, vertical transmission, or less frequently, blood transfusions or reused sharp objects. It is common among patients with HIV infection and the converse is also true. Syphilis is a disease with devastating effects if untreated. Although effective and low-cost treatment is available, syphilis continues to be a public health problem due to lack of awareness [1].

\section{CASE REPORTS}

\section{Case 1}

18 year old male presented with single painless ulcer over penis since 2 weeks. He gave history of sexual contact with multiple partners since 3 months. There were no other associated signs and symptoms.

On examination multiple, firm, rubbery, non tender lymphnodes were enlarged bilaterally. Single, well defined, indurated, non tender superficial ulcer with clean floor of $2 \times 3 \mathrm{~cm}$ present over prepuce. Single, indurated, nontender ulcer $2 \times 4 \mathrm{~cm}$ over coronal sulcus
(Fig. 1). "Dory flap sign" was present.Diagnosis was confirmed by VDRL (1:32). Patient was treated successfully with inj. Benzathine penicillin 2.4 million units and Tab. Azithromycin lgm.Counseling was done.

\section{Case 2}

28 year old male patient presented with multiple painful genital ulcers of 3 weeks duration. He gives history of genital ulcers in wife 5 months back which healed on treatment. He gives history of sexual contact with multiple partners.

On examination multiple well defined erosions over the glans with clean erythematous floor was seen. Single well defined erosion with erythematous floor which was indurated and non tender over the frenulum (Fig. 2a). Multiple linear fissures with whitish maceration present over the prepuce (Fig. 2b). Inguinal lymphnodes were bilaterally enlarged and non tender. Blood VDRL was reactive in 1:32 dilutions. HIV was also reactive. A diagnosis of secondary syphilis with candidal balanoposthitis with HIV. Patient was treated with inj. Benzathine penicillin 2.4 million units andTab. Azithromycinlgm.Systemic and topical antifungals was also given. Counseling was done. Patient was referred to ART center for further management. 


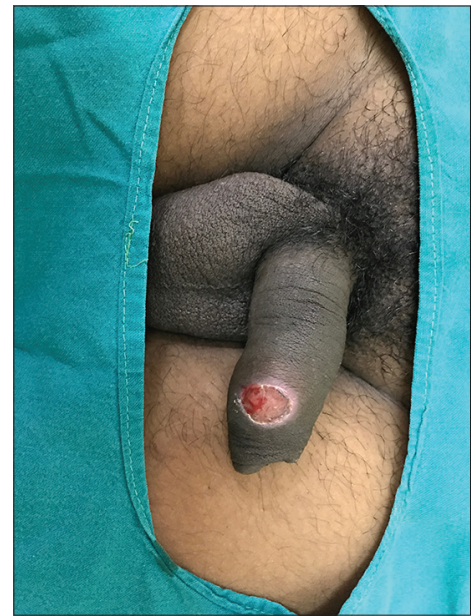

Figure1: Classical Hunterian chancre.

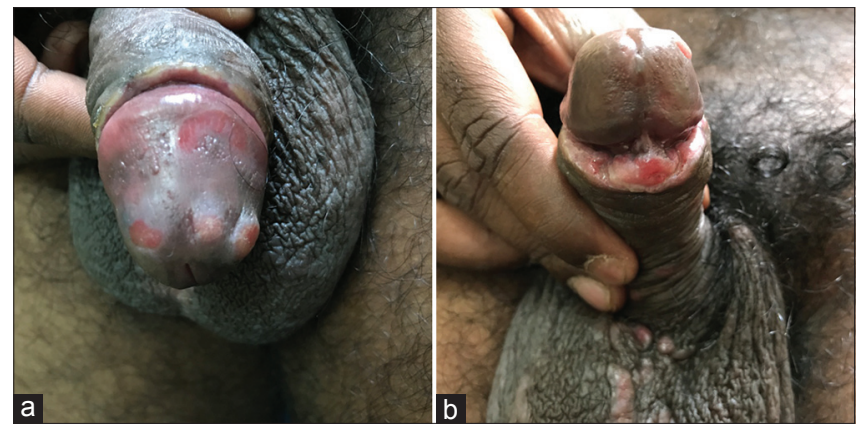

Figure $2 \mathrm{a}$ and $\mathbf{2 b}$ : A retropositive case with syphilis showing multiple erosions over glans.

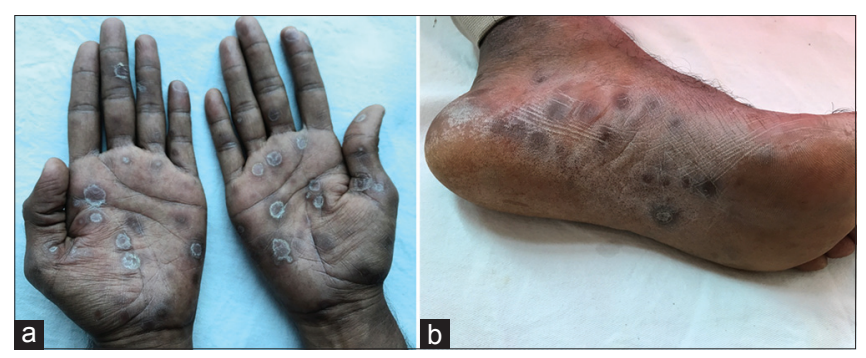

Figure 3a and 3b: Palmoplantar lesions in a retropositive patient with secondary syphilis.

\section{Case 3}

25 year old male patient presented with annular dark raised lesions over both palms and soles since 20 days. Not associated with itching or pain. History of sexual contact with multiple partners of same sex since 2 years. Last contact was 6 months back. History of significant weight loss in last 6months.On examination bilateral inguinal, cervical, epitrochlear lymphnodes were enlarged and nontender. Multiple, well defined, polysized, annular hyperpigmented plaques with minimal scaling over both palms and soles (Figs. 3a and 3b). "Buschke Ollendorff sign" was present.
Multiple erosions of $0.5 \times 1$ present over shaft of penis. Multiple, smooth, pearly white umbilicated papules over penis and perianal area. Diagnosis was confirmed by VDRL (1:32) and HIV was also reactive. A diagnosis of secondary syphilis with molluscum contagiosum with HIV was made. He was treated with Inj. Benzathine penicillin 2.4million units given IM weekly once for 3 weeks. Tab. Azithromycin lg given. Needling was done for Molluscum Contagiosum. Patient was referred to ART center for further management.

\section{DISCUSSION}

Syphilis is a chronic systemic infectious disease caused by T. pallidum. It may affect any organ in the body during its course and may result in life threatening consequences that occur in the cardiovascular and nervous systems. It is distinguished by florid manifestations on one hand and years of asymptomatic latency on the other hand. Transmissible to offspring, and treatable to the point of presumptive cure [2]. Syphilis has many uncommon presentations and requires high level of suspicion. The presentation differs in different stages such as primary, secondary, early latent and late latent.

Syphilis and HIV infection are common among patients with HIV infection and the converse is also true. Syphilis produces genital lesions or inflammatory response (macrophages and T cells) that enhance HIV replication. Strong epidemiologic association is observed between HIV and Syphilis. Several unusual manifestations of syphilis observed in the presence of concurrent HIV infection are increased severity of clinical manifestations, rapid progression of syphilis to the tertiary stage within a few weeks or months of initial infection, Sero-nonreactivity of Serological test for syphilis in the presence of an active secondary stage can occur when associated with HIV thus making the dark field examination or skin biopsy essential for the diagnosis of syphilis and relapse in spite of adequate treatment hence aggressive therapy appears mandatory at the initial presentation of syphilis with HIV infection [3]. Syphilis should also be suspected in high risk patients presenting a variety of atypical syndromes such as neurologic symptoms, uveitis or cholestatic hepatitis, especially if palmoplantar lesions are present [4].

\section{CONCLUSION}

Syphilis is rarely seen in our present day clinical scenario. This case series is being reported to highlight 
www.odermatol.com

the classical and varied presentation of syphilis and its possible association with HIV.

\section{Consent}

The examination of the patient was conducted according to the Declaration of Helsinki principles.

\section{REFERENCES}

1. Strieder ER, Leon JE, Carvalho YR, Kaminagakura E. Oral syphilis: report of three cases and characterization of the inflammatory cells. Ann Diag Pathol. 2015;19:76-80.
2. CDC. Sexually transmitted diseases treatment guidelines, 2010. MMWR. 2010;59(RR-12).

3. Shilaih M. Factors associated with syphilis incidence in the HIVInfected in the era of highly active antiretrovirals." Ed. Perbinder Grewal. Medicine 96.2 (2017): e5849. PMC. Web. 15 Oct. 2018.

4. Senecal XM, Barkati S, Bouffard D, Martel-Laferrière V. A Secondary syphilis rash with scaly target lesions. Oxf Med Case Reports. 2018;2018:omx089.

Copyright by Aryambika Krishnan, et al. This is an open access article distributed under the terms of the Creative Commons Attribution License, which permits unrestricted use, distribution, and reproduction in any medium, provided the original author and source are credited.

Source of Support: Nil, Conflict of Interest: None declared. 